a commercial technique of IB to detect TIF1 $\gamma$ (Euroimmun), however, commercial kits sometimes elaborate antigens without using whole protein structure. To date, there are no studies that study the agreement between the commercial kit and homemades IB.

Objectives: 1-To compare the commercial IB results of Anti-TIF1 $\gamma$ with homemade IB.

2-To describe and compare the clinical characteristics of the patients analyzed by both techniques.

METHODS: Observational retrospective study that included adult patients with some determination of Anti-TIF1 $\gamma$ antibodies since 2014 in two tertiary-level university hospitals. We collected demographic and clinical data of all patients. Taking into account their diagnoses we grouped them into: Cancer Associated Myositis (CAM), DM without cancer, Systemic Autoimmune Diseases (SAD) non-DM and other diagnoses. Subsequently, the clinical agreement with the IB results of Anti-TIF1 $\gamma$ performed by commercial (clB) and homemade (hIB) technique were analyzed. A p value $<0.05$ was considered statistically significant.

RESULTS: 48 patients were recruited (77.1\% women). 13 had diagnosis of CAM, 19 of DM without cancer, 7 of other non-DM EAS, and 9 had other diagnoses.

Of all DM (CAM and DM without cancer), 12 were positive for both techniques. 5 presented discordant results $(4 \mathrm{hIB}$ positive with negative clB and $1 \mathrm{clB}$ positive with negative hIB). 15 DM were negative for the two techniques, of which $8 / 15$ had other antibodies associated with Autoimmune Myopathy (3 NXP-2, 1 Mi2, 1 MDA5, 1 SAE, 1 Jo1 and 1 Ro52). When we analyzed patients with CAM with both techniques, 10/13 presented positive hIB vs 8/13 clB positive. DM without cancer, 6/19 had positive hIB vs 5/19 clB positive. The sensitivity and specificity of each test was evaluated in CAM and DM without cancer, being $61.5 \%$ and $73.7 \%$ respectively for clB $(p=0.071)$, compared to $76.9 \%$ and $68.4 \%$ for hIB ( $p=0.029$ ).

In non-DM SAD group, 1 patient had positive determination for both techniques, 3 had positive clB with negative hIB, and $3 \mathrm{clB}$ negative with positive hIB. We also found other antibodies in 6/7 patients with non-DM SAD. The 9 patients with other diagnoses were: 1 positive for both techniques and 8 positive for $\mathrm{ClB}$ and negative for hIB.

CONCLUSIONS: Homemade IB with $155 \mathrm{kDa}$ recombinant protein was superior to clB to detect TIF $\gamma$ in our serie of patients with CAM. This could have clinical implications in screening and early detection of neoplasia that affect false negative by clB.

A positive $\mathrm{clB}$ in patients not suspected as a SAD were negative for hIB in the major part of cases.

It would be recommended to check the commercial IB using homemade IB, due to its known clinical-therapeutic implications.

Disclosure of Interests: Ana Milena Millán Arciniegas: None declared, M. ANGELES MARTINEZ: None declared, ANAIS MARISCAL: None declared, ANDRES BAUCELLS: None declared, Leticia Alserawan: None declared, Ernesto Trallero: None declared, Cesar Díaz-Torné: None declared, Ana Laiz Consultant for: Lilly, Novartis, AbbVvie, MSD, UCB and Janssen, Speakers bureau: Lilly, Novartis, Abvvie, MSD, UCB and Janssen, Berta Magallares: None declared, Patricia Moya: None declared, HyeSang Park: None declared, Andrea García-Guillén: None declared, Sycille Jeria: None declared, DAVID LOBO: None declared, Albert Gil: None declared, Coral Gozalvez: None declared, Susana P. Fernandez-Sanchez: None declared, Laura Martínez-Martínez: None declared, Esther Moga: None declared, Hector Corominas: None declared, Cándido Juarez: None declared, Ivan Castellví Consultant for: I received fees less than 5000USD as a consultant for Kern and Actelion, Paid instructor for: I received fees less than 2000USD as a instructor for Boehringer -Ingelheim, Novartis and Gebro, Speakers bureau: ND, Albert Selva-O'Callaghan: None declared DOI: 10.1136/annrheumdis-2019-eular.6954

\section{AB0672 RENAL INVOLVEMENT IN PATIENTS WITH DERMATOMYOSITIS AND POLYMYOSITIS}

Simeon Monov ${ }^{1}$, Daniela Monova ${ }^{2}$, Russka Shumnalieva $^{1}$, Elena Miloshova ${ }^{2}$.

${ }^{1}$ Medical University - Sofia, Department of Rheumatology, Sofia, Bulgaria;

${ }^{2}$ Medical Institute, Medical University - Sofia, Department of Internal Diseases, Sofia, Bulgaria

Background: Dermatomyositis (DM) and polymyositis (PM) are characterized by moderate to severe muscle weakness and inflammatory lesions in the muscle. They lead to frequent, and, in some cases, life-threatening extramuscular (lung, cardiac, neurologic, kidney) complications.

Objectives: The aim of this study was to determine the incidence, the severity, the course and outcome of renal diseases in patients with DM or PM.
Methods: We identified 84 patients (68 female, 16 male) with the diagnosis of DM and 23 (14 female, 9 male) with PM. Diagnosis of DM and PM was based on the ENMC classification. The mean follow-up was 5,6 $\pm 3,8$ years. Evaluation of extramuscular involvement was mainly performed in the presence of suggestive clinical signs. Autoantibodies were detected by ELISA. Acute kidney injury (AKI) was defined as an acute doubling of serum creatinine level. Chronic kidney disease (CKD) was defined if an estimated glomerular filtration rate (eGFR) $<60 \mathrm{~mL} / \mathrm{min}$ on at least 2 measurements 3 months apart.

Results: Of the 107 patients, 26 were found to have suffered varying degree of renal involvement. All the 26 patients $(22$ patients with DM and 4 with PM) had varying degree of proteinuria and haematuria. Renal involvement consisted of $\mathrm{AKI}$ in 8 patients and $\mathrm{CKD}$ in 18 patients. Among patients with $\mathrm{AKI}, 6(75 \%)$ progressed to $\mathrm{CKD}$, including 2 patients who reached end-stage renal disease (ESRD). Acute tubular necrosis with renal failure developed in 3 patients with $P M$ and in 4 patients with DM. In descriptive analysis, male sex, cardiovascular risk factors, the severity of muscle injury, heart involvement, and initial proteinuria $(>0.3 \mathrm{~g} / \mathrm{d})$ were associated with AKI. In multivariate survival analysis, only age at disease onset, male sex, history of cardiovascular events, and a previous episode of AKI were identified as risks factors of CKD. Although the proteinuria and haematuria responded to therapy (except in 2 cases), the creatinine clearance remained decreased during the treatment. Kidney biopsy was performed in 16 patients and showed chronic tubulointerstitial nephritis ( 7 cases), minimal change disease (2 cases), focal segmental glomerulosclerosis lesions (3 cases), IgA nephropathy ( 2 cases), membranous nephropathy (1 case), and amyloid deposits (1 case).

Conclusion: Our data clearly indicate that DM and PM are associated with a wide range of renal disorders. Renal damage was noted in $24,3 \%$ of all included patients. AKI occurred in 7,48\% of cases and CKD developed in $16,82 \%$ of the patients. AKI and CKD contribute to an increased morbidity in these patients. AKI and CKD are probably not a direct manifestation of PM or DM, but rather result from drug and myoglobin-induced renal damage. However, $\mathrm{AKI}$ and $\mathrm{CKD}$ contribute to increased morbidity in these patients

Disclosure of Interests: None declared

DOI: 10.1136/annrheumdis-2019-eular.1525

\section{AB0673 CHARACTERISTICS OF THE CAPILLAROSCOPY PATTERN IN SYSTEMIC AUTOIMMUNES DISEASES}

Atusa movasat Hajhkan, Ana Isabel Turrión, Henry Moruno, Cristina Bohórquez, Ana Pérez Gómez, Eduardo Cuende, Fernando Albarrán, Ana Sánchez Atrio, Lucía Ruiz, Paula Pretel, Laura Barrio Nogal, Valentina Emperiale, Adrián Abbasi, Melchor Álvarez de Mon. hospital principe de asturias, alcala de henares, madrid, Spain

Background: Nailfold capillaroscopy (CP) is a simple, safe, low cost and non-invasive screenig test and together with the study of autoantibodies, is useful in the diagnosis of systemic sclerosis (SSc). It demonstrates certain specific patterns which are characterized by the presence of dilated capillaries, haemorrhages and avascular areas. Other systemic autoimmune diseases (SAD) do not have a specific pattern, but certain alterations in the morphology of the capillaries such as increased tortuosity have been described in SLE. In other diseases such as mixed connective tissue disease (MCTD), inflammatory myopathies and overlap syndromes, findings may be different to the pathological findings consistent with scleroderma pattern.

Objectives: Description of nailfold capillaroscopic findings in different SAD. Methods: The study included 255 patients referred for $\mathrm{CP}$ that was performed in 8 fingers, and always by the same observer. The following findings were considered as "scleroderma pattern": Local or global capillary loss (> 20\%), haemorrhages: two or more in at least two fingers and enlarged capillaries: two or more capillary with double caliber or more in at least two different fingers. Abundant evidence of tortuosity (> $20 \%$ ) as ringlets tangles and no evidence of disorders described above was considered nonspecific, tortuous pattern or suspects SAD. Statistical analysis was performed with SPSS 19.0 program.

Results: CP was performed in 255 patients, 31 males (12\%) and 224 $(88 \%)$ women. 23 patients $(9 \%)$ had a diagnosis of SSc, 6 patients $(2 \%)$ of SLE, other $6(2 \%)$ of Sjögren's syndrome, 7 (3\%) of MCTD, $4(1.5 \%)$ of inflammatory myopathy and $2(0.8 \%)$ of overlap syndrome. 18 patients (7\%) had positive ANA and Raynaud without another data suggestive of SAD, 76 patinents with primary Raynaud (30\%) and 66 patients (26\%) with a miscellany of other diseases such as antiphospholipid syndrome, vasculitis, fibromyalgia and psoriatic arthritis. Also, 29 patients (12.4\%) were sent for performing CP for suspected systemic sclerosis and 16 
patients $(6.3 \%)$ presented with only positive anti-centromere. CP was normal in 191 patients, while in 45 patients scleroderma pattern was observed and in 19 patients nonspecific pattern suggestive of SAD was seen. Capillaroscopic findings: in each of the 23 patients diagnosed with SSc, 21 patients had scleroderma pattern. Of the 6 patients with SLE, $\mathrm{CP}$ was normal in 4 patients, while one patient had abundant tortuosity. Likewise, the 6 patients with Sjögren's syndrome, only one patient had a nonspecific pattern suggestive of SAD. Of the 7 patients with MCTD, four had normal CP, two had a scleroderma pattern and one had tortuous pattern. The 2 patients with overlap (myopathy-scleroderma and Lupus-scleroderma) both had a pathological CP with scleroderma pattern. Conclusion: Our study demonstrates that the evidence of scleroderma pattern is very specific of SSc. However, in certain diseases such as MCTD, overlap syndromes and inflammatory myopathies, we can also see this pattern. In other SAD, CP is usually normal or may show some nonspecific changes.

References: The contribution of capillaroscopy to the differential diagnosis of connective autoimmune diseases. Best Practice \& Research Clinical Rheumatology Vol. 21, No. 6, pp. 1093-1108, 2007

Disclosure of Interests: None declared

DOI: 10.1136/annrheumdis-2019-eular.4133

\section{AB0674 RETROSPECTIVE STUDY OF A COHORT OF PATIENTS WITH SYSTEMIC SCLEROSIS IN A TERTIARY CARE HOSPITAL}

David Moyano Bueno ${ }^{1}$, Luis Gómez-Lechón ${ }^{2}$, Olga Martínez González ${ }^{2}$, Ana Isabel Turrión ${ }^{2}$, Carlos A. Montilla-Morales ${ }^{2}$, Cristina Hidalgo ${ }^{2} .{ }^{1}$ Complejo $^{2}$ Asistencial Universitario de Salamanca, Dermatology, Salamanca, Spain; ${ }^{2}$ Complejo Asistencial Universitario de Salamanca, Rheumatology, Salamanca, Spain

Background: Systemic sclerosis (SS) is an autoimmune disease of unknown etiology, characterized by the presence of fibrosis and vasculopathy in skin and multiple internal organs such as the lungs, the kidneys and the digestive tract. The course of the disease is unpredictable and could remain relatively stable or have a rapid evolution. Multiple studies have been carried out to determine the clinical characteristics and survival prognosis on SS patients.

Objectives: To analyze the demographic characteristics, clinical features, treatment and prognosis in a SS disease cohort.

Methods: We performed an observational and retrospective study of patients whit SS. The patients had been attended by the Rheumatology department of a tertiary care hospital. We collected demographic, clinical and analytical variables, as well as treatment and prognosis. We classified the disease employing the LeRoy and Medsger, VEDOSS criteria and 2013 ACR/EULAR criteria.

Results: Of our 43 patients, $36(83.7 \%)$ were female and only $7(16.3 \%)$ were male. The average age was 60.4 years (SD 15.6), the average age at diagnosis was 53.3 years (SD 17.6) and the mean time of evolution of the disease was 7.9 years (SD 6.3). Of all the patients, 3 patients $(6.9 \%)$ died, the mean age at death being 53.6 years (SD 23.7) and the mean time from diagnosis to death of 19 years (SD 10.1). The most frequently ocurring presentation was limited SS (with 18 patients $(41.9 \%)$, followed by preescleroderma with 14 patients (32.6\%), diffuse SS with 6 patients $(14 \%)$ and escleroderma sine escleroderma with 2 patients $(4.7 \%)$. Three patients $(7 \%)$ were labeled with MCTD. SS was associated with other autoimmune diseases in $20 \%$ of patients. Five $(11.6 \%)$ patients developed neoplasms throughout the course of the study. The rest of the clinical characteristics are listed in Table 1 and 2, as well as the strength of association of these with the type of SS, calculated using chi square and Fischer test.

Conclusion: Worthy of noting in our cohort is the absence of scleroderma in more than $40 \%$ of our patients, probably because the new criteria have allowed us to diagnose the disease at an earlier stage and also due to the scarce frequency of puffy fingers with respect to other larger series. Digestive involvement was the most frequent visceral manifestation, followed by pulmonary manifestations, specifically interstitial lung disease (ILD). Despite the small sample size, lung disease was significantly associated with the two forms of systemic sclerosis, but not with the antibody pattern. Both the ILD and the pulmonary arterial hypertension (PAH) were more frequent in patients with SSd. Mortality in all cases was due to interstitial lung involvement. As is frequently described, SS is associated with other systemic autoimmune diseases, constituting an overlap syndrome.

\section{REFERENCES}

[1] Cañete Crespillo, J. (2008). Manual SER de las enfermedades reumáticas. Madrid: Editorial Médica Panamericana.

[2] Dougherty, D., Kwakkenbos, L., Carrier, M., Salazar, G., Assassi, S. Baron, M., Bartlett, S., Furst, D., Gottesman, K., van den Hoogen, F., Malcarne, V., Mouthon, L., Nielson, W., Poiraudeau, S., Sauvé, M., Boire, G. Bruns, A., Chung, L., Denton, C., Dunne, J., Fortin, P., Frech, T., Gill, A. Gordon, J., Herrick, A., Hinchcliff, M., Hudson, M., Johnson, S., Jones, N., Kafaja, S., Larché, M., Manning, J., Pope, J., Spiera, R., Steen, V., Sutton E., Thorne, C., Wilcox, P., Thombs, B. and Mayes, M. (2018). The Scleroderma Patient-Centered Intervention Network Cohort: baseline clinical features and comparison with other large scleroderma cohorts. Rheumatology, 57(9), pp.1623-1631.

[3] CLINICAL FEATURES AND PROGNOSTIC FACTORS FOR SYSTEMIC SCLEROSIS RELATED INTERSTITIAL LUNG DISEASE. (2018). Respirology, 23, pp.155-155.

Disclosure of Interests: None declared

DOI: 10.1136/annrheumdis-2019-eular.7361

\section{$\mathrm{AB} 0675$ \\ DECREASED CAPILLARY DENSITY IS ASSOCIATED WITH DECREASED HAND MOBILITY IN SYSTEMIC SCLEROSIS}

Gabriella Nagy, Antonietta Kovács, Dalma Komjati, Renata Szijártó, Tunde Minier, Gabor Kumanovics, László Czirják, Cecília Varjú. University of Pécs, Department of Rheumatology and Immunology, Pécs, Hungary

Background: Nailfold videocapillaroscopy (NVC) is a simple method to evaluate capillary density and morphology. Systemic sclerosis (SSc) is characterized with decreased hand mobility and joint contractures evolving in hands in early disease stage. Movement ability (MA) of hands can be measured with different methods including Hand Mobility Index in Scleroderma (HAMIS), hand anatomic index (HAI), delta finger-to-palm index ( $\triangle \mathrm{FTP})$, and number of joints with decreased range of motion (joint contractures-JC).

Objectives: To examine correlation between capillary density and MA of hands in patients with SSc.

Methods: 136 patients with SSc underwent detailed examination of MA and capillary density measured with videocapillaroscop. 70 patients had limited cutaneous SSc (ICSSc) and 66 had diffuse cutaneous SSc (dcSSc). 48 patients had $\leq 5$ years of disease duration (early SSc) and 88 patients had disease duration $>5$ years (late SSc) calculated from the first non-Raynaud symptoms.

Results: DcSSc patients had significantly higher HAMIS score than IcSSc patients $(4.5 / 1.5 ; 6.5 /$ vs. $2 / 0.5 ; 4.5 / ; p<0.01)$. HAMIS score showed negative correlation with capillary density in the entire cohort (rho: -0.269 , $p<0.01$ ) and in dcSSc patients (rho:-0.487; $p<0.0001$ ), but not in IcSSc ones.

HAI showed a significantly higher value in IcSSc patients than in dcSSc patients $(3.79 / 3.18 ; 4.39 / v s .3 .19 / 2.64 ; 3.76 /, \quad p<0.01)$. Significant positive correlation between $\mathrm{HAl}$ and capillary density was found in dcSSc patients (rho: $0.377, p<0.01$ )

DcSSc patients showed significantly lower $\triangle$ FTP value than IcSSc patients $(73.88 / 65.63 ; 87.00 /$ vs. $82.81 / 73.63 ; 90.5 / ; p<0.05)$. Positive correlation was found between capillary density and $\triangle \mathrm{FTP}$ in the entire cohort, in dcSSc patients even if dcSSc was examined separately as early dcSSc and late dcSSc. No significant correlation was found between capillary density and $\triangle \mathrm{FTP}$ in IcSSc patients.

The number of JC was significantly higher in dcSSc patients than in IcSSc patients $(16 / 0 ; 28 /$ vs. 0/0;11/; $p<0.01)$, and no statistically significant difference was found between early SSc patients and late SSc patients. Strong negative correlation was found between JC count and capillary density in the entire cohort (rho -0.376; $\mathrm{p}<0.0001$ ), in dcSSc patients (rho $-0.501 ; p<0.0001$ ), in early SSc patients (rho-0.583; $p<0.0001$ ) and late SSc patients (rho $-0,262 \mathrm{p}<0.05$ ), but not in IcSSc patients.

Conclusion: Decreased capillary density correlated with decreased hand mobility and higher number of JCs. Tissue ischemia may play role in the development of joint contractures and it might be a contributing factor to decreased hand mobility impairment in SSc.

\section{REFERENCES}

[1] Torok KS, Baker NA, Lucas M, et al. Reliability and validity of the delta finger-to-palm (FTP), a new measure of finger range of motion in systemic sclerosis. Clin Exp Rheumatol. 2010;28(2)(Suppl 58):S28-S36.

[2] Sandqvist G, Nilsson JA, Wuttge DM, Hesselstrand R. Development of a modified hand mobility in scleroderma (HAMIS) test and its potential as an 